Fortnightly Review

\title{
Management of hip osteoarthritis
}

\author{
Paul Dieppe
}

Osteoarthritis of the hip is a common disorder of white (but not black) people with a roughly equal incidence in both sexes. Although more common in elderly people, it may start at any age from the second or third decade. Most cases are idiopathic, even among younger people, though a minority are secondary to a congenital or childhood hip disorder (acetabular dysplasia, congenital dislocation, slipped epiphysis, or Perthe's disease) or to an inflammatory arthropathy. ${ }^{1}$ Other risk factors for osteoarthritis may include inequalities in leg length and certain occupations or patterns of use of the hip: for example, there is a strong association between farming and hip osteoarthritis in European men. ${ }^{2}$ The condition has a chronic, variable course and often causes considerable pain and disability. Good management depends on the doctor's ability to diagnose the condition early, appreciate factors that may affect the prognosis or complicate the disease, and make effective use of the many treatment methods available.

\section{Presentation, diagnosis, and prognosis}

The start of symptomatic hip osteoarthritis is usually insidious, although in a few cases pain starts abruptly.

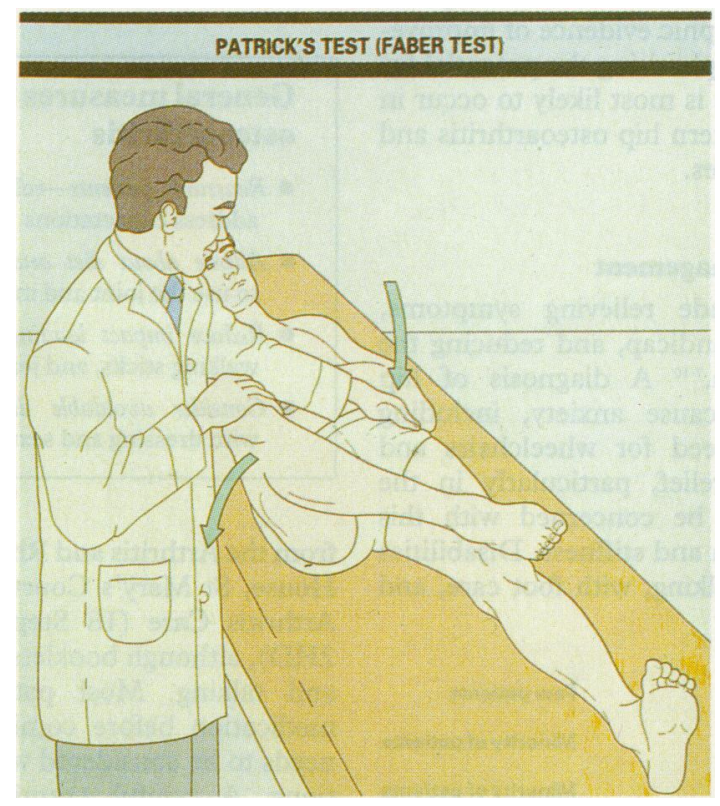

Rheumatology Unit, Department of Medicine, Bristol Royal Infirmary, Bristol BS2 8HW Paul Dieppe, $A R C$ professor *

$B M f 1995 ; 311: 853-7$
FIG 1-Patrick's manoeuvre (FABER test) to test for evidence of arthritis in hip. The flexed, abducted hip is slowly lowered while the pelvis is held down on the opposite crest: in early hip osteoarthritis the movement is restricted and painful. (Internal rotation of hip with both hip and knee flexed to $90^{\circ} \mathrm{C}$ is an alternative manoeuvre.) Range of motion of the two hips should be compared as internal rotation is first movement to be restricted by hip disease. Reproduced from Dieppe with permission of publishers

\begin{tabular}{l} 
Summary points \\
\hline - Hip osteoarthritis is a common cause of \\
regional pain, but care must be taken when \\
making the diagnosis \\
- Patients should be encouraged to live as \\
normal a life as possible: a healthy lifestyle \\
(keeping slim and active) is also good for joints \\
- Common treatable difficulties that can arise \\
from hip osteoarthritis include problems with \\
dressing, sexual difficulties, and inadequate foot \\
care \\
- Shock absorbing shoe insoles and walking \\
sticks can be of great benefit, while physio- \\
therapy and hydrotherapy should be considered \\
for more severe cases \\
- If drugs are used simple analgesics such as \\
paracetamol should be considered before non- \\
steroidal anti-inflammatory drugs \\
- Joint replacement should be considered for \\
patients with severe pain or disability and if the \\
disease has a major impact on work or social \\
activities
\end{tabular}

Joint pathology can precede symptoms by years, and the start of pain may be associated with minor trauma to an already diseased joint. The pain can vary greatly in both its site and nature, sometimes making early diagnosis difficult: it may be felt in the area of the buttock, groin, thigh, or knee and varies in character from a dull ache to sharp stabbing pains. The discomfort is generally related to activity and exercise may induce bouts of pain that last for several hours. In advanced disease severe pain may be present at night or during rest. Stiffness of the hip is usual, particularly after inactivity, and can be the presenting featurepatients complaining of difficulty putting on socks and shoes, for example.

Early physical signs include restriction of internal rotation and abduction of the affected hip, with pain at the end of the range (fig 1 ). The diagnosis can usually be confirmed by radiography. A joint space width of $2.5 \mathrm{~mm}$ or less indicates substantial loss of cartilage in the hip joint, and osteophytes, subchondral bone sclerosis, or cysts are usually present (fig 2 ). ${ }^{3}$ Radiographs are not always necessary, however, and can be misleading. For example, an elderly patient may present with "hip" pain due to a periarticular disorder, but the radiograph may show mild osteoarthritis, leading to an erroneous diagnosis. The relative insensitivity of radiography and discordance between 

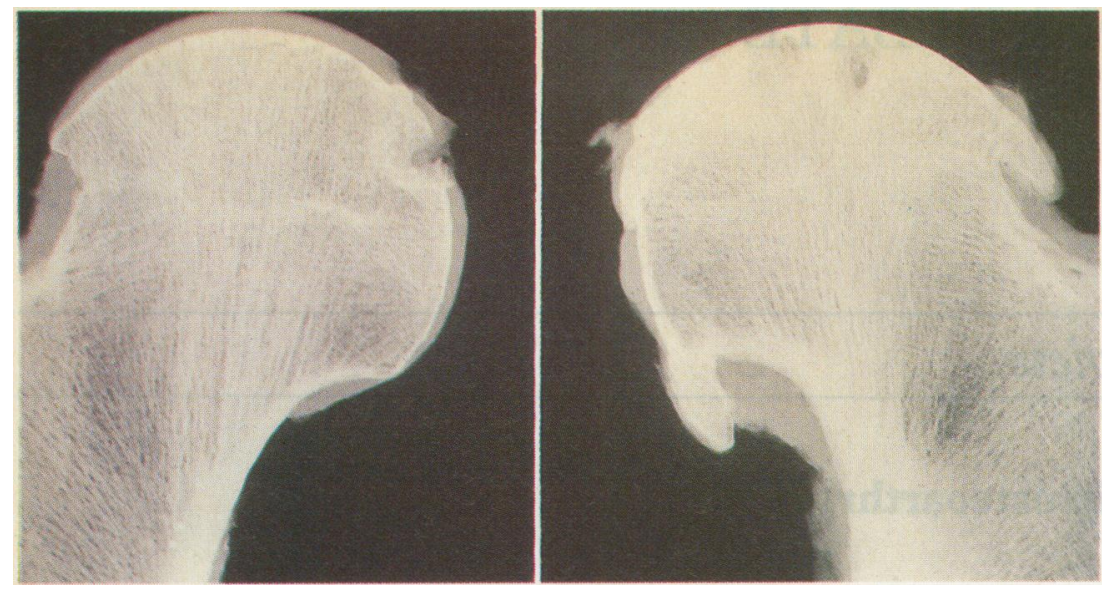

FIG 2-Slab radiographs of excised hips with osteoarthritis: in early disease (left) focal loss of articular cartilage occurs with early formation of marginal osteophytes; in advanced disease (right) there is extensive loss of cartilage, remodelling of subchondral bone with associated cysts and sclerosis, and extensive formation of osteophytes. Reproduced from Dieppe ${ }^{7}$ with permission of publishers

structural changes and symptoms are major problems in the diagnosis and assessment of all forms of likely to be missed or delayed when discomfort presents in the buttock or is referred to the knee joint and in rare cases of posterior hip joint osteoarthritis (which may not be apparent in an anteroposterior radiography but only on a lateral or oblique view). Trochanteric or ileopsoas bursitis is often misdiagosteoarthritis. ${ }^{4}$ The diagnosis of hip disease is most nosed as hip osteoarthritis, and apophyseal joint disease may cause similar symptoms.

After presentation, many cases remain relatively stable for many years, but the long term outcome is poor for most patients. Patients who require surgery for hip osteoarthritis often describe a relatively short period (1-2 years) of deterioration and severe symptoms. This and other data suggest that the condition may progress as phases of active disease interrupted by periods of stability. It is extremely difficult to provide individual patients with an accurate prognosis, although some features associated with a relatively good or poor outcome have been identified. Elderly patients and those with severe pain or discrepancies in leg length are probably more likely to show rapid progression of the disease, as are those with radiographic evidence of a superolateral subluxation of the hip or a paucity of bone response. ${ }^{5}$ However, up to $5 \%$ of advanced cases of hip osteoarthritis can improve spontaneously, with radiographic evidence of improvement as well as less pain, emphasising the potential for repair in osteoarthritis. ${ }^{6}$ This is most likely to occur in patients with concentric pattern hip osteoarthritis and extensive radiographic changes.

\section{Goals and categories of management}

Management goals include relieving symptoms, minimising disability and handicap, and reducing the risk of disease progression. ${ }^{7-10} \mathrm{~A}$ diagnosis of hip osteoarthritis is likely to cause anxiety, including concerns about eventual need for wheelchairs and major surgery. Symptom relief, particularly in the early stages, may need to be concerned with this anxiety as much as with pain and stiffness. Disabilities may include difficulty in walking, with foot care, and

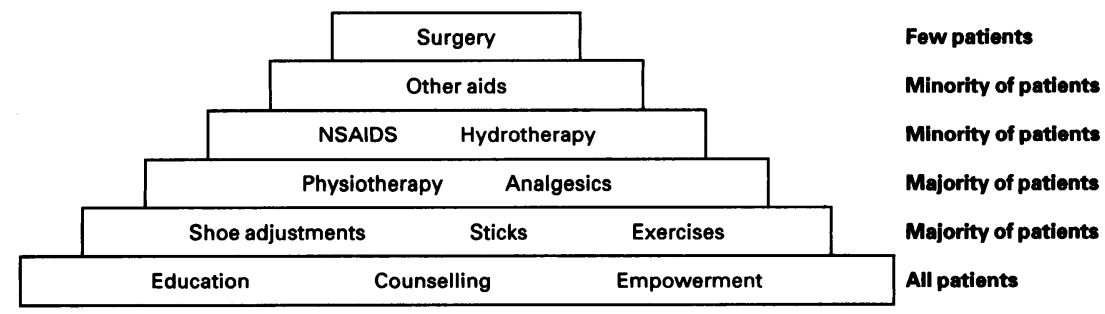

FIG 3- "Pyramidal" approach to management of hip osteoarthritis. All patients should have access to interventions at base of pyramid, but only a few will need those at the apex with dressing as well as sexual problems. The degree of handicap will depend on the debilitating effects of chronic pain as well as the degree of disability and social circumstances. These variables, combined with the wide age range of patients, give rise to great heterogenicity in the needs of those affected. Use of the most appropriate interventions at the right time requires great skill and extensive understanding of the patients and their needs.

The management of hip osteoarthritis can be conveniently split into four categories: general measures, which should be used in all cases; consideration of associated disorders and complications; specific medical and physical interventions of value to most people with established hip osteoarthritis, and surgical treatment of advanced disease. Medical and surgical

\begin{tabular}{|ll|}
\hline \multicolumn{2}{|c|}{ Goals and categories of management } \\
Goals & Categories \\
Relieve symptoms & General measures (all \\
Minimise disability and & patients) \\
handicap & Specific drugs and physical \\
Reduce risk of progression & therapy \\
& Treating complications \\
& Surgical salvage \\
\hline
\end{tabular}

interventions carry risk, whereas hip osteoarthritis is a relatively benign condition that is not life threatening and can remain stable (or even improve) with only mild pain or disability over many years. For these reasons, drugs and surgical options should not be considered in early or mild cases and a "pyramidal" approach to management is appropriate (fig 3 ). ${ }^{7-9}$

\section{GENERAL MEASURES}

Education and empowerment-Most people with osteoarthritis want to know why they have it, what will happen to them, and what they can do about it. Negative attitudes and dismal prognosis are obviously inappropriate. Giving people the feeling that they have some control and "de-medicalising" as well as demystifying the condition are often appropriate strategies. Various educational pamphlets are available

\section{General measures for treating hip osteoarthritis}

- Reassure patients-relieve anxiety and empower, address expectations

- Advise about diet and exercise-encourage patients to use the joint and maintain motion and strength

- Reduce impact loading by means of shoe insoles, walking sticks, and joint protection

- Consider avoidable disabilities such as difficulties with dressing and sexual problems

from the Arthritis and Rheumatism Council (Copeman House, St Mary's Court, Chesterfield S41 7TD) and Arthritis Care (18 Stephenson Way, London NW1 2HD), although booklets are no substitute for listening and talking. Most patients will have started self medication before coming to their doctor, and this needs to be considered while listening to their expectations. A careful examination of the joint can be reassuring, and giving advice about what patients can do for themselves is of immense value. ${ }^{10}$

Rest and exercise-Patients are often frightened that use will "wear out" a damaged hip joint and need "permission" to use it. There is some evidence that lack of use may be deleterious and that an affected joint 


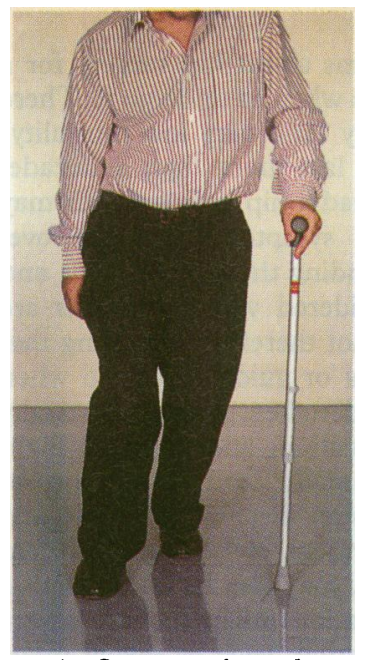

FIG 4 -Correct use of properly adjusted walking stick. Stick should be held on opposite side to affected hip to take weight when step is taken on painful side; it should reach to brim of pelvis; and it should have good ferrule and comfortable handle. Reproduced from Dieppe $e^{7}$ with permission of publishers needs regular loading to help maintain its integrity. However, prolonged or heavy activity may cause a lot of pain. Most patients learn an appropriate balanceinterspersing periods of activity with rest and not pushing the hip too far ("little and often"). It is important to maintain a full range of hip movement if possible. In addition to specific exercises, recreations such as swimming or cycling may help. Contact sports and activities such as jogging, which can cause repetitive high impact loading of the hip, are probably best avoided, but the general benefits of moderate exercise can be accompanied by striking reductions in pain and disability in people with established knee osteoarthritis. ${ }^{11}$

Diet and weight-There is no evidence for the involvement of any dietary factor in the aetiopathogenesis of hip osteoarthritis. However, it is possible that obesity may accelerate progression or cause more pain. Patients who are overweight should be advised to lose weight for the health of their hips as well as their hearts.

Shoes and sticks-Many people with arthritis of the spine or legs are more comfortable wearing trainers or other shoes with good shock absorbing properties than they are in regular footwear. Sorbithane and other shock absorbing insoles (which are available in sports and shoe shops) may be helpful. A simple walking stick can make a big difference, reducing loading on a hip by $20-30 \%$, but attention must be given to its length, characteristics, and use. In most cases it will be most beneficial if it is held on the unaffected side of the body and if it comes to the top of the pelvis and has a good ferrule and a comfortable handle (fig 4).

Socks and sex-Common disabilities associated with hip osteoarthritis include difficulty putting on socks or stockings, tying shoelaces, cutting toenails, and walking up and down steps and stairs and painful sexual intercourse. ${ }^{12}$ These problems are often due to loss of range of hip movement as well as pain on movement. They can often be treated by physiotherapy and exercises to help increase the range of movement. Alternatively, simple aids or advice-such as dressing sticks and counselling about positions for sexual intercourse, may make a huge difference. Foot care may need special attention.

\section{SPECIFIC DRUGS AND PHYSICAL THERAPY} Physiotherapy and hydrotherapy

There is evidence that maintaining a full range of joint motion, and strong muscles around an affected joint may reduce the risk of the disease progressing as well as greatly decreasing pain and disability. The importance of these measures cannot be overstressed, although it is not yet clear how to effect this policy to large numbers of people over several years, in an economic way.

Referral to a physiotherapist is indicated if hip osteoarthritis is causing substantial pain and disability in spite of application of the general therapeutic measures outlined above. Consequences of hip osteoarthritis that the physiotherapist may be able to treat

\section{Specific physical therapy and drug treatment for hip osteoarthritis}

- Physiotherapy to improve range of movement and muscle strength and help relieve pain

- Aids such as walking aids, dressing sticks, bath seats, etc

- Drugs-start with a simple analgesic, consider nonsteroidal anti-inflammatory drugs later if safe to do so

- Other measures-consider complementary techniques to help relieve severe pain effectively include secondary muscle weakness (the cause of the Trendelenburg sign) and loss of movement, which are major sources of disability. Hydrotherapy, as well as exercise on land, may be of great value in restoring a better range of joint motion through a combination of pain relief, muscle relaxation, and stretching exercises. There is, however, a surprising paucity of objective data documenting either the efficacy or cost effectiveness of physiotherapy for osteoarthritis of the hip. ${ }^{13}$

\section{Drug treatment}

New, potentially disease modifying drugs are being developed for the treatment of osteoarthritis. At present, however, only symptom modifying drugs are available. Because the hip is a deep joint and relatively inaccessible, local treatments such as rubefacients are of less value than at other peripheral sites affected by osteoarthritis. Most people expect to use systemic treatment for pain, and most doctors prescribe oral drugs for osteoarthritis. Simple fast acting analgesics such as paracetamol or low dose ibuprofen are probably most appropriate for most patients. In early or mild disease they should be used on demand and not regularly. People can learn to take a couple of tablets before doing something that they know is likely to aggravate the hip.

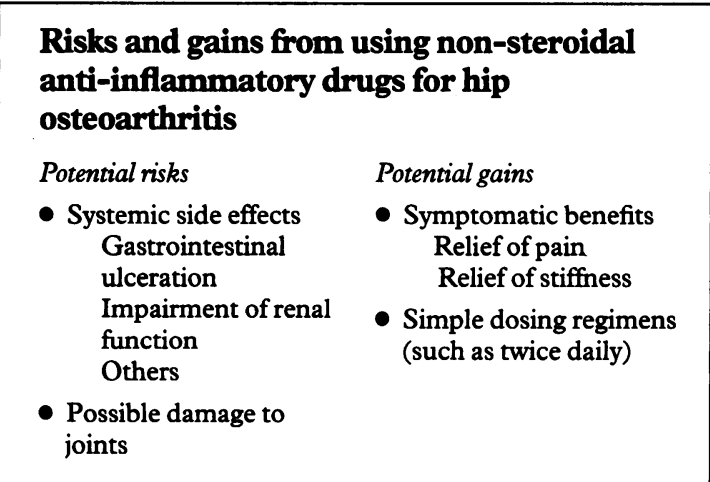

The relative efficacy and toxicity of analgesics and non-steroidal anti-inflammatory drugs in hip osteoarthritis is a subject of debate for which inadequate data are available. ${ }^{14} 15$ If the simple, on demand, approach to using paracetamol or low dose ibuprofen is insufficient, regular use or stronger analgesics might be considered. Data from investigations on patients with knee osteoarthritis suggest that some (but by no means all) patients find a non-steroidal anti-inflammatory drug more effective than an analgesic. ${ }^{16}{ }^{17}$ Clinical experience suggests that the same is probably true of the hip. If such drugs are to be used, safety aspects should be of paramount importance in prescribing for benign conditions common in elderly people-such as hip osteoarthritis. Indomethacin should probably be avoided as there is some evidence that it may accelerate damage. ${ }^{18} 19$ Non-steroidal anti-inflammatory drugs should be given for short periods of a few weeks only, to help overcome exacerbation of pain rather than as a lifetime prescription. Some of the major concerns with non-steroidal anti-inflammatory drugs include the risk of gastrointestinal haemorrhage or perfusion, and renal disease, both of which are particularly likely in elderly patients. ${ }^{2021}$

\section{Other non-surgical measures for severe pain or disability}

The best treatment for severe pain or disability is surgical, but this is not always an option and may be delayed for some months. In such circumstances other measures-including local corticosteroid injections, acupuncture, or transcutaneous electrical nerve 
stimulation-might be considered for pain relief and the skills of occupational therapists and social workers might be invoked to help manage disability. Walking aids and house adaptations might also be necessary.

\section{ASSOCIATED CONDITIONS AND COMPLICATIONS}

Trochanteric bursitis-Hip osteoarthritis and its associated muscle weakness can result in secondary discomfort from the fascia lata or lead to trochanteric bursitis. Severe asymmetrical point tenderness directly over the greater trochanter of the affected hip suggests the diagnosis. The condition often responds well to a local injection of a long lasting corticosteroid. ${ }^{22}$ If this is mixed with local anaesthetic the immediate effects on pain and disability can be assessed.

\section{Associated conditions and complications of hip osteoarthritis}

- Bursitis-trochanteric or iliopsoas bursitis can mimic or complicate hip osteoarthritis

- Unequal leg lengths can be the cause or result of hip osteoarthritis

- Spinal osteoarthritis can mimic, coexist with, or complicate hip osteoarthritis

- Intra-articular complications can include haemorrhage, synovitis, collapse, fractures, and necrosis

\section{Indications for surgery}

One of the main problems about hip surgery for a person with osteoarthritis is when to undergo it. There is a perioperative morbidity (and very low mortality) and a prosthesis may not last more than a decade. Furthermore, as noted already, hip osteoarthritis may not progress quickly and symptoms can improve. Clearly, many factors, including the patient's role and aspirations, must be considered when decisions are made about surgery. It is not therefore surprising that there are no agreed criteria or guidelines as to when hip surgery should be undertaken. Pain is generally regarded as the main indication, and intrusive night pain in particular will usually lead a surgeon to recommend surgery. Severe disability, particularly if it is threatening employment or having important psychosocial consequences, may also be an indication for surgery. Relative indication might include severe effects on a particular function, such as sexual intercourse. The general adage that surgery is indicated if a patient cannot work, sleep, walk, or play gives some idea of the issues other than severity of pain that should be considered.

Age is also an important factor. Prostheses are more likely to fail in young active people who demand near normal use, but this has to be balanced against the needs and aspirations of younger patients, whose demands for a good quality of life in their "prime of life" may be well justified. The decision whether to undergo surgery is the patient's decision, and it should be made to meet the realistic functional aspirations of the individual. Thus, two patients with similar severity of pain and functional impairment may have quite different needs: a young executive whose working life, social aspirations, and golfing handicap are being eroded may demand a new hip, whereas an elderly person whose life revolves around his or her house, memories, and friends who visit may be content with a walking stick and an occasional paracetamol.

\section{Perioperative care}

The preparation for surgery should include a check of general health, with particular attention to the presence of any infections or intercurrent drug treatment, and advice about the likely outcome, including the limitations of surgery. After the operation patients will need advice about avoiding movements that cause hip abduction in the first few months and other ways of protecting the new joint while maintaining independent and gradually increasing activity.

I thank the Arthritis and Rheumatism Council for financial support and Professor Ian Learmonth for his advice.

1 Felson DT. The epidemiology of hip and knee osteoarthritis. Epidemiol Rev 1988;10:1-28.

2 Croft P, Coggon D, Cruddas M, Cooper C. Osteoarthritis of the hip, an occupational disease in farmers. BMF 1992;304:1269-71.

3 Croft $P$, Cooper C, Wickham C, Coggan D. Defining osteoarthritis of the hip for epidemiological studies. Am ₹ Epidemiol 1990;132:514-22.

4 Jones A, Doherty M. ABC of rheumatology osteoarthritis. BMF 1995;310: 457-60.

5 Ledingham J, Dawson S, Preston B, Milligan G, Doherty M. Radiographic progression of hospital referred osteoarthritis of the hip. Ann Rheum Dis progression of hosp

SURGERY
Hip replacement has proved one of the major advances in medicine this century. In spite of current concerns about later loosening of the artificial joint, as well as infrequent early complications, it is clearly of great and lasting value to most of the many thousands of people operated on each year. Such is the success of the total hip replacement, that other optionsincluding osteotomy or fusion-are rarely considered in Britain or North America. However, osteotomy remains a viable alternative to joint replacement for the relief of pain, particularly when economic factors are dominant, and is still widely used in many parts of the world..$^{23}$
6 Radin EL, Burr D. Joints can heal. Semin Arthritis Rheum 1984;13:293-308.

7 Dieppe P. Osteoarthritis management. In: Klippel J, Dieppe P, eds. Rheumatology. London: Mosby, 1994:781-8.

8 Dieppe PA. Management of osteoarthritis of the hip and knee joints. Current osteoarthritis of the hip and knee. I Roy Coll Phys (London) 1993;27:391-6.

10 The management of osteoarthritis. Northallerton: Primary Care Rheumatology Society, 1994.

11 Kovar PA, Allegrante JP, McKenzie R, Peterson M, Gutin B, Charlson M. Supervised fitness walking in patients with osteoarthritis of the knee. Ann Intern Med 1992;116:529-34.

12 Curry HLF. Osteoarthritis of the hip joint and sexual function. Ann Rheum Dis 1970;29:488-91.

13 Puett DW, Griffin MR. Published trials of non-medical and noninvasive therapies for hip and knee osteoarthritis. Ann Intern Med 1994;121:133-40.

14 Dieppe PA, Frankel SJ, Toth B. Is research into the treatment of osteoarthritis Opinion in Rheumatology 1993;5:487-93.

9 Scott DL. Guidelines for the diagnosis, investigation and management of 
with non-steroidal anti-inflammatory drugs misdirected? Lancet 1993;341: 353-4.

15 Liang MH, Fortin P. Management of osteoarthritis of the hip and knee (editorial). N Engl f Med 1991;325:125-7.

16 Bradley JD, Brandt KD, Katz BP, Kalasinski LA, Ryan SI. Comparison of an anti-inflammatory dose of ibuprofen, an analgesic dose of ibuprofen, and acting NEngloph 1991 325:87-91.

17 March L, Irwig L, Schwarz J, Simpson J, Chock C, Brooks P. N of 1 trials comparing non-steroidal anti-inflammatory drug with paracetamol in comparing non-steroidal anti-inflam

18 Newman NM, Ling RSM. Acetabular bone destruction related to nonsteroidal anti-inflammatory drugs. Lancet 1985;ii:11-4.

19 Rashad S, Revell P, Hemingway A, Low F, Rainsford K, Walker F. Effect of non-steroidal anti-inflammatory drugs on the course of osteoarthritis. Lancet 1989;2:111-3.

20 Bateman DN, Kennedy JG. Non-steroidal anti-inflammatory drugs and elderly patients. $B M F$ 1995;310:817-8.

21 Jobanputra $P$, Nuki G. Non-steroidal anti-inflammatory drugs in the treatment of osteoarthritis. Curr Opin Rheumatol 1994;6:433-9.

22 Schapira D, Nahir M, Scharf Y. Trochanteric bursitis: a common clinical problem. Arch Phys Med Rehabil 1986;67:815-7.

23 Amstrutz HC, Kin WC. Osteoarthritis of the hip. In: Moskowitz R, Howell D, Goldberg V, Mankin H, eds. Osteoarthritis. 2nd ed. Philadelphia: Saunders, 1992:314-42.

(Accepted 24 fuly 1995)

\title{
Review of cases of nosocomial Lassa fever in Nigeria: the high price of poor medical practice
}

\author{
S P Fisher-Hoch, O Tomori, A Nasidi, G I Perez-Oronoz, Y Fakile, L Hutwagner, J B McCormick
}

Special Pathogens Branch, Division of Viral and Rickettsial Diseases, National Center for Infectious Diseases, Centers for Disfase Control and Prevention,

Atlanta, GA 30333, USA

S P Fisher-Hoch, deputy branch chief

G Perez-Oronoz, medical technologist

Y Fakile, visiting scientist

J B McCormick, branch chief

Biostatistic Branch, Division of Bacterial and Mycotic Diseases,

National Center for

Infectious Diseases

L Hutswagner, biostatistician

Department of Virology, College of Medicine, University of Ibadan, Ibadan, Nigeria

O Tomori, professor

Federal Epidemiological Division, Federal Ministry of Health, Lagos, Nigeria A Gneissoid, chief, vaccine laboratory

Correspondence to: Dr S P Fisher-Hoch, Aga Khan University Medical School, PO Box 3500, Stadium Road, Karachi 74800, Pakistan. jbm@halycon.com

\section{Abstract}

Objective-To investigate two hospital outbreaks of Lassa fever in southern central Nigeria.

Setting-Hospitals and clinics in urban and rural areas of Imo State, Nigeria.

Design-Medical records were reviewed in hospitals and clinics in both areas. Patients with presumed and laboratory confirmed Lassa fever were identified and contracts traced. Hospital staff, patients, and local residents were questioned, records were carefully reviewed, and serum samples were taken. Serum samples were assayed for antibody specific to Lassa virus, and isolates of Lassa virus were obtained.

Results-Among 34 patients with Lassa fever, including 20 patients, six nurses, two surgeons, one physician, and the son of a patient, there were 22 deaths ( $65 \%$ fatality rate). Eleven cases were laboratory confirmed, five by isolation of virus. Most patients had been exposed in hospitals (attack rate in patients in one hospital 55\%). Both outbreak hospitals were inadequately equipped and staffed, with poor medical practice. Compelling, indirect evidence revealed that parenteral drug rounds with sharing of syringes, conducted by minimally educated and supervised staff, fuelled the epidemic among patients. Staff were subsequently infected during emergency surgery and while caring for nosocomially infected patients.

Conclusion-This outbreak illustrates the high price exacted by the practice of modern medicine, particularly use of parenteral injections and surgery, without due attention to good medical practice. High priority must be given to education of medical staff in developing countries and to guidelines for safe operation of clinics and hospitals. Failure to do so will have far reaching, costly, and ultimately devastating consequences.

In early March 1989 three doctors from two different hospitals in Imo State, south central Nigeria (fig 1), died of suspected Lassa fever at the University of Nigeria Teaching Hospital, Enugu, within a period of 11 days. One doctor owned a small clinic (hospital A) in Aboh Mbaise (population 500000 ), a densely populated agricultural area $20 \mathrm{~km}$ north of the Imo state capital, Owerri. The other two were a surgeon and the physician-owner of a hospital (hospital B) close to the crowded marketplace of Aba (population 1000000 ) $60 \mathrm{~km}$ south east of Owerri. Lassa fever is a severe haemorrhagic disease endemic in southern Nigeria, as in most of west Africa. ${ }^{12}$ It is caused by an arenavirus that persistently infects a west African rodent, Mastomys natalensis.

\section{Investigations of the outbreaks}

The investigating team was assembled and travelled to the two hospitals following requests for help from physicians at the University of Nigeria Teaching Hospital and the Nigerian Ministry of Health. The two hospitals were deserted, and the investigations were conducted amid high levels of fear, suspicion, and rumour. It was not possible to obtain all the desired evidence, but we were able to identify cases of Lassa fever retrospectively in both epidemics. Most surviving hospital staff were traced and blood samples taken, but many surviving patients were unwilling to cooperate. We gathered information on these and missing staff members from records and interviews. We conducted a careful analysis of all available medical records and reviewed all inpatient deaths from December 1988 in outbreak hospitals and all other hospitals in Aboh Mbaise, Aba, and Owerri. Presumptive Lassa fever was defined by history of prolonged fever (more than five days), sore throat, proteinuria, oedema, bleeding or convulsions, and lack of response to antimicrobial and antimalarial agents between 1 January and 31 March $1989 .{ }^{3}$ Laboratory confirmation was by either or both the isolation of Lassa virus or the detection of IgG and IgM by immunofluorescent antibody assays against standard Lassa virus and isolates from this epidemic. ${ }^{4}$

Interviews were completed on 935 individuals and 814 serum samples were obtained. In Aboh Mbaise

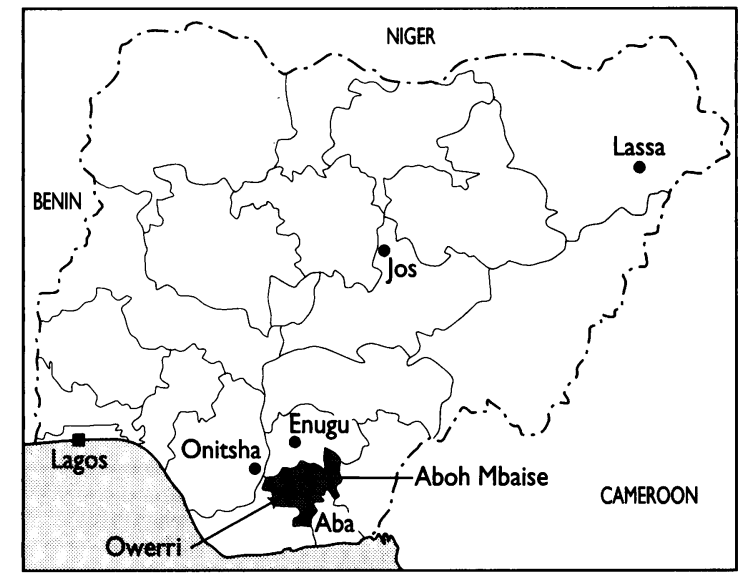

FIG 1-Location of the two outbreaks sites and previous reports of Lassa fever in Nigeria. Imo State is shaded 